\title{
A case report of sharp recanalization in a hemodialysis patient with severe occlusion of both superior and inferior vena cave
}

\author{
Zaibo Liao', Li Zhou ${ }^{2}$, Xing Zheng ${ }^{3}$, Xi Shen ${ }^{2}$, Tianlei Cui ${ }^{2,4}$ \\ ${ }^{1}$ The Center of Gerontology and Geriatrics, West China Hospital, Sichuan University/West China School of Nursing, Sichuan University, Chengdu, \\ China; ${ }^{2}$ Division of Nephrology, Department of Internal Medicine, West China Hospital of Sichuan University, Chengdu, China; Department of \\ Internal medicine, Dang Xiong People's Hospital, Lhasa, China; ${ }^{4}$ Division of Intervention, West China Hospital of Sichuan University, Chengdu, \\ China \\ Correspondence to: Tianlei Cui. Division of Nephrology, Department of Internal Medicine, West China Hospital of Sichuan University, Chengdu, \\ China. Email: tianleicui@163.com.
}

\begin{abstract}
We present the case of a 53-year-old woman with a history of maintenance hemodialysis through arteriovenous fistula, CUFF catheter (cuffed tunneled catheter) and artificial vascular graft successively. Some signs of superior vena cava syndrome have presented including chronic edema in the face and left arm and varicose veins. Both CT (computed tomography) and angiography showed narrowing and occlusion in multiple veins, especially the right innominate vein, superior vena cava, inferior vena cava, left jugular vein, and bilateral common iliac veins. The first attempt at recanalization of the AVG (arteriovenous graft) failed due to severe occlusion of central venous. Finally, the patient was treated with a minimally invasive surgical approach involving percutaneous direct superior vena cava puncture driven by a single bend and vascular snare that were placed in the right atrium via hepatic vein percutaneous direct puncture under a double C arm angiographic device. The initial attempt failed with RUPS 100 (Cook, Chicago, USA) and was complicated by pericardial tamponade. The second attempt succeeded and the catheter was placed without hindrance and edema or varicose veins and its patency has remained for over 18 months following the intervention. It is the first successful case of sharp recanalization in combined superior and inferior vena cava syndromes with long-segment occlusion. This creative approach to providing vascular access offers a novel way to address refractory lesions in central veins.
\end{abstract}

Keywords: Superior and inferior vena cava; sharp recanalization; hemodialysis; case report

Submitted Dec 29, 2020. Accepted for publication Mar 11, 2021.

doi: 10.21037/apm-21-189

View this article at: http://dx.doi.org/10.21037/apm-21-189

\section{Introduction}

While superior and inferior vena cava occlusion syndromes are common, severe types may result in long-term complications, which threaten the lives of hemodialysis patients. Rebuilding vascular access is difficult. There are some studies have reported the techniques including sharp recanalization and puncture superior vena cave. However, the patients in these reports suffered from vascular occlusion either superior vena cava or brachiocephalic vein individually, while inferior vena cava is often spared entirely $(1,2)$. But there are no reports of rebuilding vascular access in cases of multiple central vein occlusion involving the superior and inferior vena cava with the conditions occur bilaterally and the presence of severe edema. The novel method of placing a vascular snare in the right atrium via the hepatic vein, puncturing the superior vena cava directly, and then recanalizing occlusion of the superior vena cava was considered in this case as the patient's condition was life-threatening. We describe the first successful case of sharp recanalization in combined superior and inferior vena cava syndromes with long-segment occlusion. We present the following article in accordance with the CARE reporting checklist (available at http://dx.doi.org/10.21037/ apm-21-189). 


\section{Case presentation}

A 53-year-old woman with a history of end stage renal disease (ESRD) secondary to glomerulonephritis had multiple failed arteriovenous and CUFF catheter dialysis accesses. Her case was also complicated by the presence of chronic edema in the face and left arm, varicose veins, and multiple central venous occlusions (Figure 1A). She had twice undergone surgery that placed an arteriovenous fistula (AVF) on her left arm, had a CUFF catheter in the right jugular vein for 5 years and in the right femoral vein for 4 years, and had an artificial vascular graft (AVG) in the left femoral vein for 2 years. A temporary catheter in the right femoral vein had been placed for the previous 3 months because of dysfunction of the AVG. CT scan and angiography confirmed narrowing and occlusion of multiple veins, especially the right innominate, superior and inferior vena cava, left jugular, and bilateral common iliac veins (Figure 1B,C,D,E).

The first attempt at recanalization of the AVG failed due to severe occlusion of the inferior vena cava and bilateral common iliac vein, despite the use of balloon-expanding and stent placement. A second attempt took place 1 day later using percutaneous direct puncture of the superior vena cava guided by a double $\mathrm{C}$ arm angiographic device (Figure $1 F$ ). Prior to this, single bend and vascular snare as orientations were placed in the right atrium via hepatic vein percutaneous direct puncture. The initial attempt at recanalization of long-segment complete occlusion of the superior vena cava failed with RUPS 100 and was complicated by pericardial tamponade (Figure $1 G$ ). However, after 30 minutes of observation, a secondary recanalization with RUPS 100 and balloon succeeded (Figure 1H,I, f). The catheter was placed without hindrance and edema or varicose veins and its patency has remained for over 18 months following the intervention.

All procedures performed in studies involving human participants were in accordance with the ethical standards of the institutional and/or national research committee(s) and with the Helsinki Declaration (as revised in 2013). Written informed consent was obtained from the patient's guardian for publication of this study and any accompanying images.

\section{Discussion}

Occlusions of the inferior vena cava and superior vena cava/ right atrium junction meant that in this case, puncture and catheterization was not desirable via the hepatic vein or inferior vena cava. As a life saving measure, orientation of the right atrium via the hepatic vein and sharp recanalization of the superior vena cava were attempted as a novel approach. Factors for procedural success included: position, length of vein, collusion, elastic recoiling, and method of recanalization (3-5). The placement of catheters in the right jugular and femoral veins, together with the high blood flow of the AVG, were the main causes for occlusion as well as superior and inferior vena cava syndromes and represented a serious challenge to establishing vascular access. Fortunately, a $6 \mathrm{~cm}$ segment of vein remained available between the right femoral and right external iliac veins, which made a temporary catheter for briefly providing blood flow of $150 \mathrm{~mL} / \mathrm{min}$ and allowed daily hemodialysis prior to the operation.

The consulting nephrologist cautioned that the femoral vein was used more than the right jugular vein, and that the catheter should be transferred to the AVF as soon as possible. AVF first and AVF prepared in advance were recommended methods to prevent superior and inferior vena cava syndromes and CT angiography should be applied before AVG implantation to verify there was no stenosis of the central venous.

While blunt recanalization may be safer for slight or medium central venous occlusion, because of the severe and long-segment lesions present in this case, it was considered inadequate. Traditional thoracic surgery was also considered inappropriate because of the major trauma involved and associated high mortality (6). Sharp recanalization was considered in this case, despite the increased risk of complication compared with the blunt method. There are few reports detailing the successful treatment of multiple central vein occlusions with superior and inferior vena cava occlusions. In our case, because of the occlusions of the inferior vena cava and at the junction of the superior vena cava and right atrium, puncture and catheterization was not desirable via the hepatic vein or inferior vena cava. As the patient's condition was life-threatening, orientation in the right atrium via the hepatic vein and sharp recanalization of the superior vena cava was attempted as a novel approach.

Recently, using different methods and tools to cross the chronically occluded segments have been called sharp recanalization. The most common method for sharp recanalization uses a stiff guide wire, PTCD puncture needle, and RUPS100 and transseptal needle $(1,7)$. Common complications in this type of surgical procedure include pain, bleeding associated with vein varicosities 

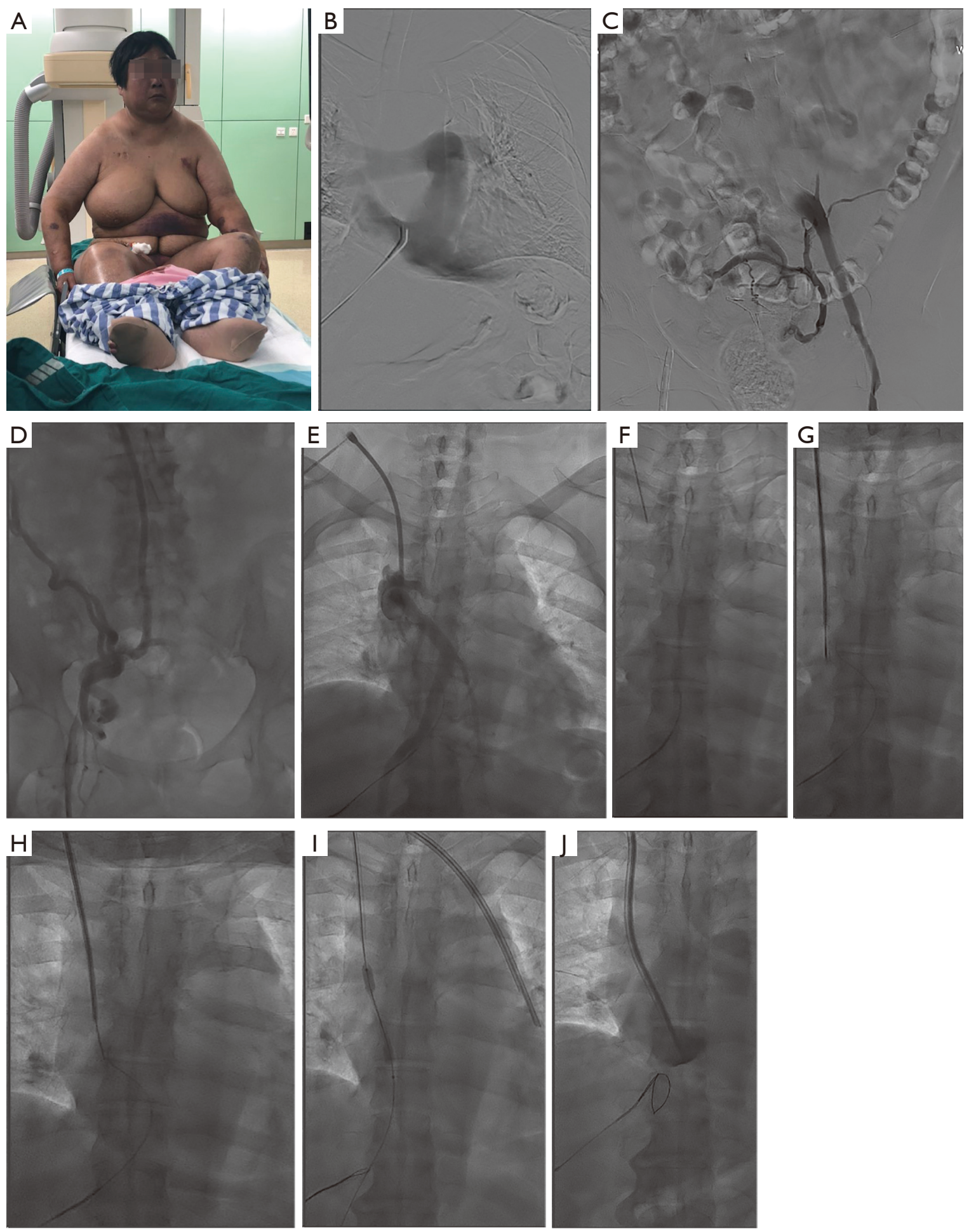

Figure 1 The patient at prior to the procedure and anteroposterior fluoroscopic images during the procedure. (A) Chronic edema is seen in the face, arms and legs, and varicose veins on the arm, thoracic and abdominal wall; (B,C,D,E) computed tomographic scan and angiography confirmed narrowing and occlusion of the superior and inferior vena cava, right innominate, left jugular, and bilateral common iliac veins; (F) percutaneous direct puncture of the superior vena cava was applied; (G) a failed first attempt at reopening with RUPS 100; (H,I,J) a successful secondary attempt with RUPS 100 and balloon. 
caused by compensatory changes in the operation area, infection, and damage to nearby tissue and organs (8). More severe complications include malignant arrhythmia, hydrothorax, hydropericardium, and especially pericardial tamponade, for which there is no efficient hemostasis method, posing a serious threat to life.

Balloon-expanding and stent had been used on the AVG and bilateral common iliac veins in our patient. However, because of occlusions in the inferior vena cava, the superior vena cava pathway was considered. DSA guiding of the anterior and lateral position via the occluded stub of the right innominate vein and direct puncture of the stub of the superior vena cava proved successful. An $8 \mathrm{~F}$ sheath was placed and the tip of the sheath was entered into the stub of the superior vena cava, the shape of which was observed by radiography. An RUPS100 was then chosen because of the long segment occlusion. In addition, tips of the RUPS100 could be shaped according to the distorted superior vena cava and position of the right atrium.

Although the patient suffered pericardial tamponade during the procedure, after 30 minutes of non-active bleeding of pericardium, a second attempt resulted in success, with the catheter being placed with its tip to the right atrium and with good blood flow. It is advisable to adopt the technique combining RUPS-100 and hepatic vein direct puncture in a life-threatening situation.

\section{Summary}

Although superior and inferior vena cava syndromes are not rare in hemodialysis patients, they are often neglected. Sharp recanalization with RUPS100 may be a successful method for relieving long-segment occlusion of the superior vena cava. While pericardial tamponade is a possible complication, in the absence of an active bleed, a second attempt might be considered.

\section{Acknowledgments}

Funding: Innovative Research on blockchain Model for Hemodialysis / Vascular Access Follow-up and Emergency Operation Appointment. "1.3.5 project for disciplines of excellence-Clinical Research Incubation Project, West China Hospital, Sichuan University".

\section{Footnote}

Reporting Checklist: The authors have completed the CARE reporting checklist. Available at http://dx.doi.org/10.21037/ apm-21-189

Conflicts of Interest: All authors have completed the ICMJE uniform disclosure form (available at http://dx.doi. org/10.21037/apm-21-189). The authors have no conflicts of interest to declare.

Etbical Statement: The authors are accountable for all aspects of the work in ensuring that questions related to the accuracy or integrity of any part of the work are appropriately investigated and resolved. All procedures performed in studies involving human participants were in accordance with the ethical standards of the institutional and/or national research committee(s) and with the Helsinki Declaration (as revised in 2013). Written informed consent was obtained from the patient's guardian for publication of this study and any accompanying images.

Open Access Statement: This is an Open Access article distributed in accordance with the Creative Commons Attribution-NonCommercial-NoDerivs 4.0 International License (CC BY-NC-ND 4.0), which permits the noncommercial replication and distribution of the article with the strict proviso that no changes or edits are made and the original work is properly cited (including links to both the formal publication through the relevant DOI and the license). See: https://creativecommons.org/licenses/by-nc-nd/4.0/.

\section{References}

1. Yang L, Yang L, Zhao Y, et al. The feasibility and safety of sharp recanalization for superior vena cava occlusion in hemodialysis patients: A retrospective cohort study. Hemodial Int 2020;24:52-60.

2. Zhao Y, Lin F, Yang L, et al. Sharp recanalization of the brachiocephalic vein occlusion through the external jugular vein in hemodialysis patients. Ann Transl Med 2020;8:640.

3. Li H, Wang SX, Wang W, et al. Thrombosis of the superior vena cava and auxiliary branches in patients with indwelling catheterization of the internal jugular vein. Chin Med J (Engl) 2009;122:692-6.

4. Schillinger F, Schillinger D, Montagnac R, et al. Post catheterisation vein stenosis in haemodialysis: comparative angiographic study of 50 subclavian and 50 internal jugular accesses. Nephrol Dial Transplant 1991;6:722-4.

5. MacRae J, Ahmed A, Johnson N, et al. Central vein stenosis: a common problem in patients on hemodialysis. 
ASAIO J 2005;51:77-81.

6. Wan Z, Lai Q, Zhou Y, et al. Efficacy and safety of a mother-child technique for recanalization of chronic central venous occlusive disease in hemodialysis patients. J Vasc Surg Venous Lymphat Disord 2020;8:558-64.

7. Arabi M, Ahmed I, Mat'hami A, et al. Sharp Central Venous Recanalization in Hemodialysis Patients: A Single-
Institution Experience. Cardiovasc Intervent Radiol 2016;39:927-34.

8. Agarwal AK, Khabiri H, Haddad NJ. Complications of Vascular Access: Superior Vena Cava Syndrome. Am J Kidney Dis 2017;69:309-13.

(English Language Editor: B. Draper)
Cite this article as: Liao Z, Zhou L, Zheng X, Shen X, Cui T. A case report of sharp recanalization in a hemodialysis patient with severe occlusion of both superior and inferior vena cave. Ann Palliat Med 2021;10(3):3495-3499. doi: 10.21037/apm-21-189 